\title{
Nuclear spin relaxation in paramagnetic solutions. Effects of large zero-field splitting in the electron spin Hamiltonian
}

\author{
Robert R. Sharp \\ Department of Chemistry, The University of Michigan, Ann Arbor, Michigan 48109
}

(Received 19 June 1990; accepted 31 July 1990)

\begin{abstract}
Expressions are derived describing nuclear spin relaxation in paramagnetic salt solutions under conditions where the electron spin Hamiltonian is dominated by a uniaxial quadratic zero-field splitting (zfs) interaction. In this situation, the electron spin vector is quantized along molecular axes rather than along the external magnetic field. By expressing the time dependence of the electron spin operators, written in the molecular coordinate frame, in the Heisenberg representation and then transforming these expressions to the laboratory coordinate system, simple closed form expressions for the paramagnetic nuclear relaxation increment have been derived. Electron-nuclear dipole-dipole and scalar relaxation mechanisms are considered. The resulting expressions parallel those of SolomonBloembergen-Morgan theory, but are valid in the zfs limit rather than the Zeeman limit. Nuclear relaxation rates in the zfs and Zeeman limits exhibit characteristic qualitative differences, some of which have been noted in earlier studies. Of particular note is the fact that the scalar contribution to $T_{1 p}^{-1}$ is much larger in the zfs than in the Zeeman limit. In most circumstances, $T_{1 p}^{-1}=T_{2 p}^{-1}$ in the zfs limit, while in the Zeeman limit, scalar relaxation usually contributes significantly only to $T_{2 p}^{-1}$. A vector model of this phenomenon is suggested. The results are valid for arbitrary values of the electron spin quantum number but they assume that electron spin relaxation is in the Redfield limit, i.e., that the correlation times of the coupling between electron spin and the lattice be short on the time scale of electron spin relaxation. This condition is probably satisfied widely when the static zfs is large.
\end{abstract}

\section{INTRODUCTION}

Spin relaxation of nuclei by paramagnetic metal ions in solution generally results from time-dependent electron-nuclear dipolar and scalar magnetic couplings as well as, in certain instances, fluctuations in the local magnetic field arising from the Curie spin magnetization. ${ }^{1}$ The latter mechanism is expected to be significant only for macromolecules when molecular reorientation is slow and when the static magnetic field is large. The former two mechanisms usually dominate; these are described by the theory of Solomon $^{2}$ and Bloembergen and Morgan. ${ }^{3}$ Limitations of the SBM theory have been discussed. ${ }^{4,5}$ Probably the most serious is its assumption that the electron spin Hamiltonian is dominated by the Zeeman interaction, that is, that zero-field splittings are negligible. This will normally be true for solutions of spin doublet species (e.g., organic radicals) and, among transition metal ions, for Mn(II) and Gd(III), for which zero-field splittings (zfs) are frequently rather small due to the absence of first-order spin-orbit coupling between the ground electronic state $\left[{ }^{6} A_{1}\right.$ for $\mathrm{Mn}$ (II) and ${ }^{8} A_{1}$ for $\mathrm{Gd}(\mathrm{III})]$ and low-lying excited states. For most other ions, the $\mathrm{zfs}$ arising from the electrostatic potential of the ligand field is much larger, and SBM theory must be applied with greater care. [The term "zero-field splitting" as used here includes level splittings that are caused by electrostatic and magnetic interactions which depend on even powers (bilinear or higher) of the electronic spin operators. These include, in the terminology of Abragam and Bleaney, ${ }^{6}$ electronic quadrupole couplings as well as, for spin $S \geqslant 2$, higher-order electrostatic interactions of the spin with the lattice. It also includes magnetic dipole couplings between individual electron spins. It does not include electron-nuclear hyperfine couplings although these, strictly speaking, also contribute to the level splittings at zero field.]

A general theory of spin relaxation in paramagnetic solutions has been developed ${ }^{7}$ starting from the Liouville equation of motion

$$
\frac{d}{d t} \rho=-i[\mathscr{H}, \rho] \equiv-i \mathscr{L} \rho
$$

of the density operator $\rho$. The stochastic Liouville equation has been applied to the calculation of line shapes in esr spec$\operatorname{tra}^{8}$ and, more recently, to the analysis of nuclear spin relaxation in paramagnetic solutions. ${ }^{5,9-15}$ The Liouville operator $\mathscr{L}$ describing an electron spin, a nuclear spin, and their couplings with each other, with the static laboratory magnetic field, and with the lattice can be written

$$
\mathscr{L}_{T}=\mathscr{L}_{I Z}+\mathscr{L}_{S Z}+\mathscr{L}_{I S \text {,dip }}+\mathscr{L}_{I S, \mathrm{sc}}+\mathscr{L}_{\text {zfs }}+\mathscr{L}_{I Q} \text {. }
$$

Successive terms describe, respectively, the nuclear Zeeman interaction, the electronic Zeeman interaction, electron-nuclear dipolar and scalar hyperfine couplings, the zero-field interaction of the electron spin with the lattice, and the electrical quadrupole interaction of the nucleus. The principal advantage of the Liouville formalism is its generality: the equations of motion can be formulated using an electron spin Hamiltonian that includes both Zeeman and zfs interactions, and it can be solved numerically under conditions 
where the electron spin system is not in the Redfield limit, i.e., when the electron spin relaxation time $\tau_{S}$ is comparable to or shorter than the characteristic correlation times of the coupling between the electron spin and the lattice. While it offers great generality, the Liouville approach has substantial disadvantages. These reside in the relatively cumbersome methodology needed and in the fact that the resulting solutions are obtained numerically. Lacking the closed form analytical expressions of SBM theory, the results are not very transparent with regard to underlying physical processes. The Liouville approach has been used to analyze nuclear relaxation in model systems, particularly $\mathrm{Ni}\left(\mathrm{H}_{2} \mathrm{O}\right)_{6}^{2+}$, ${ }^{16-18}$ for which $S=1$, and for tris- (acetylacetonato) chromium (III), ${ }^{14,19}$ for which $S=3 / 2$.

The present work is concerned with the limiting situation in which a static zfs interaction dominates the Hamiltonian of the $S$ spin so that

$$
\mathscr{H}_{\text {zfs }} \gg \mathscr{H}_{S Z}, \mathscr{H}_{I S, \text { dip }}, \mathscr{H}_{I S, \mathrm{sc}}
$$

and

$$
\mathscr{L}_{\text {zfs }} \gg \mathscr{L}_{S Z}, \mathscr{L}_{\text {IS, dip }}, \mathscr{L}_{I S, \mathrm{sc}} .
$$

In this case, the spin vector $\bar{S}$ is quantized along molecular axes. [ $S$ and $\bar{S}$ are spin quantum number and spin vector, respectively, so that $\left.|\bar{S}|^{2}=S(S+1)\right]$. The natural basis system for writing the spin Hamiltonian of $S$ is the molecular coordinate system which diagonalizes the zfs tensor. The use of this basis brings considerable simplification to the description of the time dependence of the electron spin operators. In particular, the time correlation functions of $\bar{S}$ which appear in the density matrix theory of nuclear spin relaxation, namely, $\left\langle S_{Z}(t) S_{Z}(0)\right\rangle$ and $\left\langle S_{ \pm}(t) S_{\mp}(0)\right\rangle$, can be obtained directly from the equations of motion of the spin operators in the Heisenberg representation. The resulting functions possess rather simple closed forms that are valid for general $S$. After averaging over molecular orientations, this approach leads to analytical expressions for the nuclear relaxation rates, $T_{1 p}^{-1}$ and $T_{2 p}^{-1}\left(\equiv R_{1,2 p}\right)$, that parallel the analogous results of SBM theory but are appropriate to the limit of large zfs (the zfs limit). Magnetic dipole-dipole and scalar relaxation mechanisms are considered. The approach follows that of Lindner, ${ }^{20}$ who derived limiting analytical expressions for dipole-dipole relaxation in the specific case of $S=1$. A similar, though somewhat less detailed, treatment of intermolecular (solute-solvent) nuclear spin relaxation in paramagnetic solutions has recently been given. ${ }^{21}$

The present approach does not explicitly calculate the relaxation behavior of $\bar{S}$ through a detailed solution of the equation of motion for the coupled lattice and spin systems. Rather, as in SBM theory, the electron spin relaxation times enter as parameters of the theory. In this regard, it should be noted that the definitions of electron spin relaxation times differ markedly in the zfs and Zeeman limits. In the former, relaxation occurs with respect to molecular axes, in the latter, with respect to laboratory axes. This leads to interesting differences in relaxation behavior in the two limits. Of particular note is the fact that the scalar contribution to $T_{1 p}^{-1}$ is much larger in the zfs than in the Zeeman limit. In most circumstances, $T_{1 p}^{-1}=T_{2 p}^{-1}$ in the zfs limit, while in the Zeeman limit, scalar relaxation usually contributes signifi- cantly only to $T_{2 p}^{-1}$. Also, in the zfs limit there are important characteristic differences in relaxation behavior produced by integer vs half-integer electron spin systems. The physical basis of these phenomena is discussed below.

\section{THEORETICAL}

\section{A. Magnetic dipole relaxation}

Relaxation of the nuclear spin $I$ is presumed to result from magnetic dipole coupling to the magnetic moment of an unpaired electron spin $S$ in the same molecule. $S$ is subject to a quadratic uniaxial zfs interaction, which is the dominant term of the electron spin Hamiltonian. $I$ and $S$ are separated by a vector $\overline{\mathrm{r}}$, oriented at polar angles $(\theta, \phi)$ in the molecular coordinate system which diagonalizes the zfs tensor. The molecule containing $I$ and $S$ reorients rapidly on the time scale of nuclear, but not necessarily electron, spin relaxation. The electron spin system is assumed to remain at thermal equilibrium during the relaxation processes of $I$.

The electron-nuclear dipolar Hamiltonian can be written as a scalar product of two first-rank spherical tensors ${ }^{21,22}$

$H_{\text {dip }}=\hbar \mathscr{H}_{\text {dip }}=\hbar \sum_{q} 3^{-1 / 2}(-1)^{1-q} I_{q}^{(1)} F_{-q}^{(1)}$.

The $I_{q}^{(1)}$ are the components of the first-rank spherical tensor formed by the nuclear spin operators

$$
\begin{aligned}
& I_{ \pm}^{(1)}=\mp 2^{-1 / 2} I_{ \pm}, \\
& I_{0}^{(1)}=I_{z},
\end{aligned}
$$

and $F^{(1)}$ is a first-rank spherical tensor formed from the direct product of spherical tensors of the electron spin and polar spatial variables, $F^{(1)}=\kappa r^{-3}\left\{S^{(1)} \otimes C^{(2)}\right\}^{1}$.

$$
\begin{aligned}
F_{+1}^{(1)}= & 3^{1 / 2 \cdot 5^{-1 / 2}} \kappa r^{-3}\left[\left(6^{-1 / 2}\right) S_{+1}^{(1)} C_{0}^{(2)}\right. \\
& \left.+\left(-2^{-1 / 2}\right) S_{0}^{(1)} C_{+1}^{(2)}+S_{-1}^{(1)} C_{+2}^{(2)}\right], \\
F_{0}^{(1)}= & 3^{1 / 2} \cdot 5^{-1 / 2} \kappa r^{-3}\left[( - 2 ^ { - 1 / 2 } ) \left(S_{+1}^{(1)} C_{-1}^{(2)}\right.\right. \\
& \left.\left.+S_{-1}^{(1)} C_{+1}^{(2)}\right)+(2 / 3)^{1 / 2} S_{0}^{(1)} C_{0}^{(2)}\right], \\
F_{-1}^{(1)}= & 3^{1 / 2} \cdot 5^{-1 / 2} \kappa r^{-3}\left[6^{-1 / 2} S_{-1}^{(1)} C_{0}^{(2)}\right. \\
& \left.+\left(-2^{-1 / 2}\right) S_{0}^{(1)} C_{-1}^{(2)}+S_{+1}^{(1)} C_{-2}^{(2)}\right]
\end{aligned}
$$

with

$$
\kappa=30^{1 / 2} \gamma_{I} g \beta_{0}\left(\frac{\mu_{0}}{4 \pi}\right) .
$$

These equations are in SI units; $\gamma_{I}$ is the magnetogyric ratio of spin $I, g$ is the electron $g$ factor, $\beta_{0}$ the Bohr magneton, $\mu_{0}$ is the magnetic permeability of free space, and $r$ is the $I-S$ interspin distance. The $S_{q}^{(1)}$ are defined

$$
\begin{aligned}
& S_{ \pm 1}^{(1)}=\mp 2^{-1 / 2} S_{ \pm}, \\
& S_{0}^{(1)}=S_{z},
\end{aligned}
$$

and the $C_{m}^{(2)}(\theta, \varphi)$ are Racah's normalized spherical harmonics,

$$
C_{m}^{(2)}(\theta, \varphi)=\left(\frac{4 \pi}{5}\right)^{1 / 2} Y_{2, m}(\theta, \varphi)
$$

of rank 2 and order $m . F^{(1)}$ constitutes the lattice portion of the dipolar coupling Hamiltonian. The electron spin vari- 
ables $S_{q}^{(1)}$ and the spatial variables both are assumed to remain at thermal equilibrium during the relaxation of $I$.

Using Eq. (1) for the dipolar Hamiltonian, the longitudinal nuclear relaxation rate can be written, using results of density matrix theory: ${ }^{23}$

$$
\begin{aligned}
\frac{1}{T_{1, \mathrm{dip}}}= & -2^{-1} \cdot 3^{-1} \int_{-\infty}^{\infty} d t\left[\left\langle F_{-1}^{(1)}(t) F_{+1}^{(1)}(0)\right\rangle e^{i \omega_{j} t}\right. \\
& \left.+\left\langle F_{+1}^{(1)}(t) F_{-1}^{(1)}(0)\right\rangle e^{-\omega_{1} t}\right] d t
\end{aligned}
$$

This result assumes that Redfield theory provides a valid description of nuclear spin relaxation but not necessarily of electron spin relaxation (or more specifically, that the correlation times of the interactions responsible for nuclear spin relaxation are short compared to $T_{1, I}$, while those responsible for electron spin relaxation are not necessarily short compared to $\left.\tau_{1, S}\right)$. In Eq. (6), the tensor components of the $F_{q}^{(1)}(t)$ are written in the laboratory coordinate frame. The following physical situations can be distinguished:

(1) The Zeeman limit. Both $\bar{I}$ and $\bar{S}$ are quantized in the laboratory frame by a dominant Zeeman interaction. Averaging over molecular orientations in an isotropic liquid corresponds to averaging the functions $C_{m}^{(2)}(\theta, \varphi)$ over the Euler angles. When the the spin and spatial variables are uncorrelated in time, the averages can be computed analytically, giving the results of SBM theory.

(2) The zfs limit. $\bar{I}$ is quantized by the laboratory field and $\bar{S}$ is quantized in the molecular coordinate frame. In this situation, it is most convenient to transform the components of $\bar{S}$ [given in the laboratory frame by Eq. (4)] to the molecular coordinate system using the Wigner rotational matrix elements,

$$
S_{q}^{(1)}=\sum_{q^{\prime}} \hat{S}_{q^{\prime}}^{(1)} \mathscr{D}_{q^{\prime} q}^{(1)}(\alpha, \beta, \gamma ; t) .
$$

(The karat superindex denotes vector and tensor components defined in the molecular coordinate system which diagonalizes the zfs tensor.)

(2a) Intermolecular relaxation in the zfs limit. In this situation, the spatial coupling of $\bar{S}$ and $\bar{I}$ [described by the tensor $C^{(2)}(\theta, \phi)$ and the interspin distance $\left.r\right]$ fluctuates in the laboratory frame due to molecular translational diffusion. Mathematically, the fluctuation is described by the time correlation functions

$$
\frac{C_{q}^{(2)}(\theta, \phi ; 0)}{r_{0}^{3}} \times \frac{C_{-q}^{(2)}(\theta, \phi ; t)}{r^{3}},
$$

where $(\theta, \phi)$ are polar coordinates in the laboratory frame, and $r_{0}$ is the value of $r$ at $t=0$. Calculation of these functions requires an appropriate physical model of translational diffusion. In addition to its time dependence due to translational motions, the local dipolar field of the spin $\bar{S}$ fluctuates due to molecular reorientation of the molecular axes, as described by time dependence in the Wigner matrix elements $\mathscr{D}_{q^{\prime} q}^{(1)}(\alpha, \beta, \gamma ; t)$ in Eq. (7). And finally, a third source of time dependence arises from the motion of $\bar{S}$, due both to spin precession and relaxation. This time dependence is described by the time correlation functions $\left\langle\hat{S}_{+q}^{(1)}(0) \hat{S}_{-q}^{(1)}(t)\right\rangle$, in which the spin components $\hat{S}_{q}^{(1)}(t)$ are expressed in the mo- lecular coordinate system. The forms of these correlation functions are discussed in greater detail below.

(2b) Intramolecular relaxation of $S$ in the zfs limit. In this situation also, $\bar{S}$ is quantized in the molecular frame. Thus the first-rank tensor $F_{q}^{(1)}$ in Eq. (6) is most conveniently expressed in molecular coordinates, using the Wigner matrices:

$$
F_{q}^{(1)}(t)=\sum_{q^{\prime}} \hat{F}_{q^{\prime}}^{(1)}(t) \mathscr{D}_{q^{\prime} q}^{(1)}(\alpha, \beta, \gamma ; t) .
$$

The functions $C_{q}^{(2)}(\theta, \phi)$, which describe the relative spatial orientation of the nuclear and electronic spins, are fixed in the molecular frame. Thus the time dependence of $\widehat{F}_{q^{\prime}}^{(1)}(t)$ is due solely to electron spin relaxation and is contained in the time correlation functions $\left\langle\hat{S}_{q}^{(1)}(0) \hat{S}_{-q}^{(1)}(t)\right\rangle$. The transformation matrix elements $\mathscr{D}_{q^{\prime} q}^{(1)}(\alpha, \beta, \gamma ; t)$ are time dependent due to molecular reorientation.

An explicit expression for $T_{1, \text { dip }}$ can be derived from Eqs. (1)-(8). In the following, the Euler angles \{which are the arguments of the Wigner transformation matrix elements [Eqs. (7) and (8)]\} and the polar angles $\{$ which are the arguments of the spherical harmonics [Eq. (5)]\} are omitted for the sake of brevity. Writing the functions $\left\langle F_{+1}^{(1)}(t) F_{-1}^{(1)}(0)\right\rangle e^{i \omega_{1} t}$ in the molecular frame gives

$$
\begin{aligned}
& \left\langle F_{+1}^{(1)}(t) F_{-1}^{(1)}(0)\right\rangle e^{i \omega_{1} t} \\
& =e^{i \omega_{I^{t}}}\left\langle\sum_{q^{\prime}} \hat{F}_{q^{\prime}}^{(1)}(t) \mathscr{D}_{q^{\prime}+1}^{(1)}(t) \sum_{q^{\prime \prime}} \hat{F}_{q^{\prime}}^{(1)}(0) \mathscr{D}_{q^{\prime \prime}-1}^{(1)}(0)\right\rangle \\
& =e^{i \omega_{t^{t}}} \sum_{q}\left\langle\hat{F}_{q}^{(1)}(t) \hat{F}_{-q}^{(1)}(0)\right\rangle \overline{\mathscr{D}}_{q+1}^{(1)}(t) \mathscr{D}_{-q-1}^{(1)}(0)
\end{aligned}
$$

where the orthogonality properties of the Wigner rotational matrix elements have been used to contract the double summation. Equation (9) assumes that spin and spatial functions are uncorrelated in time and that the averages can be taken separately.

The quantities $\overline{\mathscr{D}_{q+1}^{(1)}(t) \mathscr{D}_{q-1}^{(1)}(0)}$ describe reorientation of the molecular axes with respect to the laboratory frame. If the reorientational motion of the $I-S$ vector is described by the classical diffusion equation, these functions decay exponentially with respect to time, with three reorientational correlation times required in general for a complete description of a rigid molecule (corresponding to three rotational degrees of freedom of a rigid molecule). ${ }^{24}$ For simplicity, we assume that molecular reorientation is isotropic, so that

$$
\begin{aligned}
& \overline{\mathscr{D}_{q+1}^{(1)}(t) \mathscr{D}_{-q-1}^{(1)}(0)} \\
& \quad=(-1)^{q+1} \overline{\left|\mathscr{D}_{q+1}^{(1)}(0)\right|^{2}} \exp \left(-t / \tau_{R}^{(1)}\right) \\
& \quad=(-1)^{q+1} 3^{-1} \exp \left(-t / \tau_{R}^{(1)}\right) .
\end{aligned}
$$

It should be noted in Eq. (10) that the reorientational correlation time $\tau_{R}^{(1)}$ describes the motion of a first-rank tensor and thus is not the same as that which appears in formulas for dipole-dipole relaxation in the Zeeman limit, which is $\tau_{R}^{(2)}$. From classical diffusion theory, ${ }^{24}$ 


$$
\left(\tau_{R}^{(l)}\right)^{-1}=l(l+1) D_{R},
$$

where $D_{R}$ is the trace of the rotational diffusion tensor, so that $\tau_{R}^{(1)} / \tau_{R}^{(2)}=3$.

With these assumptions, the integrand of Eq. (6) can be written

$$
\begin{aligned}
= & \frac{\exp \left(-t / \tau_{R}^{(1)}\right)}{3}\left(e^{i \omega t^{t}}+e^{-i \omega, t}\right) \\
& \times \sum_{q}(-1)^{-q+1}\left\langle\hat{F}_{q}^{(1)}(t) \hat{F}_{-q}^{(1)}(0)\right\rangle .
\end{aligned}
$$

Using Eqs. (3a)-(3c),

$$
\begin{aligned}
\sum_{q}(-1)^{q-1}\left\langle\widehat{F}_{q}^{(1)}(t) \widehat{F}_{-q}^{(1)}(0)\right\rangle= & \left\langle\widehat{F}_{+1}^{(1)}(t) \hat{F}_{-1}^{(1)}(0)\right\rangle+\left\langle\widehat{F}_{-1}^{(1)}(t) \widehat{F}_{+1}^{(1)}(0)\right\rangle-\left\langle\hat{F}_{0}^{(1)}(t) \widehat{F}_{0}^{(1)}(0)\right\rangle \\
= & 3^{1} \cdot 5^{-1} \kappa^{2} r^{-6}\left\{\left\langle\hat{S}_{0}^{(1)}(t) \hat{S}_{0}^{(1)}(0)\right\rangle\left[-\left(\frac{2}{3}\right) \hat{C}_{0}^{(2)} \hat{C}_{0}^{(2)}+\widehat{C}_{-1}^{(2)} \widehat{C}_{+1}^{(2)}\right]\right\} \\
& +\left\{\left(\left\langle\hat{S}_{+1}^{(1)}(t) \hat{S}_{-1}^{(1)}(0)\right\rangle+\left\langle\hat{S}_{-1}^{(1)}(t) \hat{S}_{+1}^{(1)}(0)\right\rangle\right)\right. \\
& \left.\times\left[6^{-1} \widehat{C}_{0}^{(2)} \hat{C}_{0}^{(2)}-2^{-1} \widehat{C}_{-1}^{(2)} \hat{C}_{+1}^{(2)}+\widehat{C}_{+2}^{(2)} \widehat{C}_{-2}^{(2)}\right]\right\}
\end{aligned}
$$

The form of the time correlation functions $\left\langle\hat{S}_{q}^{(1)}(t) \hat{S}_{-q}^{(1)}(0)\right\rangle$ and the significance of electron spin relaxation times in the zfs limit vs those in the Zeeman limit deserves some comment. In the Zeeman limit, $\bar{S}$ is quantized along laboratory axes, and its relaxation times, $\tau_{S 1}$ and $\tau_{S 2}$, are defined with respect to these axes. Both quantities are (at least in principle) measurable by esr spectroscopy. For transition metal ions with electron spins $\mathbf{S} \geqslant 1$, relaxation results from thermal modulation of the zfs tensor, which provides a time-dependent perturbation to the Zeeman energy. The time dependence of the zfs results from both molecular reorientation and from vibrational relaxation of the coordination sphere of the metal ion.

In the zfs limit, $\bar{S}$ is quantized along molecular axes and undergoes its precessional motion with respect to these axes. In a uniaxial zfs, the spin Hamiltonian is

$\mathscr{H}_{\mathrm{zfs}}=\widetilde{D}\left[\left(S_{z}\right)^{2}-\frac{1}{3} S(S+1)\right]=\left(\frac{2}{3}\right)^{1 / 2} \widetilde{D} \hat{S}_{0}^{(2)} \equiv \omega_{m} \hat{S}_{0}^{(2)}$,

where $\widetilde{D}=2 \pi c D$ is the zfs in $\operatorname{rad} s^{-1}$. The time dependence of the precessional motion of $\bar{S}$ can be written in the Heisenberg representation, for arbitrary $S$, in analytical form ${ }^{21}$ :

$$
\begin{aligned}
\hat{S}_{q}^{(1)}(t) & =e^{i \mathscr{W} t(t)} \hat{S}_{q}^{(1)}(0) e^{-i \mathscr{t} t t^{t}} \\
\hat{S}_{0}^{(1)}(t) & =e^{i \omega S_{0}^{(2)} t} \hat{S}_{0}^{(1)} e^{-i \omega S_{0}^{(2)} t}=\hat{S}_{0}^{(1)} \\
\hat{S}_{ \pm 1}^{(1)}(t) & =e^{i \omega S_{0}^{(2)} t} \hat{S}_{ \pm 1}^{(1)} e^{-i \omega S_{0}^{(2)} t} \\
& =\exp \left[ \pm i \frac{\omega}{2} c_{2,0}^{+}\left(2 S_{0}^{(1)} \mp 1\right) t\right] \hat{S}_{ \pm 1}^{(1)} \\
& =\exp \left[ \pm i \widetilde{D}\left(2 S_{0}^{(1)} \mp 1\right) t\right] \hat{S}_{ \pm 1}^{(1)},
\end{aligned}
$$

where $c_{2,0}^{+}=6^{1 / 2}$ (the phase is chosen to make the raising operator real and positive). With respect to relaxation, the role of the zfs and Zeeman interactions are reversed; the Zeeman interaction provides a random perturbation to the spin Hamiltonian that is modulated by molecular reorientation, thereby relaxing the zero-order spin states of the zfs Hamiltonian. Vibrational modulation of the zfs tensor provides an additional (quite possibly dominant) relaxation mechanism. Spin relaxation times can be defined with respect to the molecular coordinate system through the time correlation functions of the components of $\hat{S}$. Assuming an exponential relaxation function (which is strictly valid in the Redfield limit for $S^{25-27}$ ), the diagonal matrix elements of the needed time-correlation functions are

$$
\begin{aligned}
& \left\langle S, m\left|\hat{S}_{0}^{(1)}(t) \hat{S}_{0}^{(1)}(0)\right| S, m\right\rangle=m^{2} \exp \left(-t / \tau_{m}^{(1)}\right), \\
& \left\langle S, m\left|\hat{S}_{-1}^{(1)}(t) \hat{S}_{+1}^{(1)}(0)\right| S, m\right\rangle=\left(-2^{-1}\right)\left|c_{S, m}^{+}\right|^{2} \exp (-i \widetilde{D}[2 m+1] t) \exp \left(-t / \tau_{m, m+1}^{(2)}\right) \\
& \left\langle S, m\left|\hat{S}_{+1}^{(1)}(t) \hat{S}_{-1}^{(1)}(0)\right| S, m\right\rangle=-2^{-1}\left|c_{S, m}^{-}\right|^{2} \exp (+i \widetilde{D}[2 m-1] t) \exp \left(-t / \tau_{m, m-1}^{(2)}\right) \\
& =-2^{-1}\left|c_{S, \mu}^{+}\right|^{2} \exp (+i \widetilde{D}[2 \mu+1] t) \exp \left(-t / \tau_{\mu+1, \mu}^{(2)}\right) \text {. }
\end{aligned}
$$

$c_{S, m}^{+}$is the raising operator of the $S$ spin manifold, its modulus given by $\left|c_{S, m}^{+}\right|^{2}=S(S+1)-m(m+1)$. In Eq. (17c), the relation $\left|c_{S, m}^{-}\right|^{2}=\left|c_{S, m-1}^{+}\right|^{2}$ has been used with the definition $\mu \equiv \mathrm{m}-1$.

Equations (17a) $-(17 c)$ describe the motion of the spin vectors through the matrix elements of the time correlation functions of the tensor components $S_{q}^{(1)}$. The precessional motion of the spin vector results from its interaction with the stationary Hamiltonian $\mathscr{H}_{\text {zfs }}$ and is described by the timedependent factors of Eqs. (15) and (16). In addition to the precessional motion, the spin operators contain time dependence due to thermal relaxation processes. These can be described formally by stochastic terms of the electron spin Hamiltonian. The major stochastic terms for $S>1 / 2$ de- 
scribe vibrational modulation of $\mathrm{zfs}$ tensor and rotational modulation of the Zeeman interaction. In Eqs. (17a)(17c), the effect of relaxation is described phenomenologically by longitudinal and transverse spin relaxation times, $\tau_{m}^{(1)}$ and $\tau_{m, m \pm 1}^{(2)}$. In the zfs limit, $\tau_{m}^{(1)}$ and $\tau_{m, m \pm 1}^{(2)}$ are not in principle measurable by esr, since spin relaxation occurs with respect to the molecular coordinate frame, not the laboratory frame. In the following, I will not distinguish between them.

Evaluating the traces $\left\langle\hat{S}_{q}^{(1)}(t) \hat{S}_{-q}^{(1)}(0)\right\rangle$ $\equiv \operatorname{Tr}\left\{\hat{S}_{q}^{(1)}(t) \hat{S}_{-q}^{(1)}(0)\right\}$ in the high-temperature limit for $S$, we have

$$
\begin{aligned}
& \left\langle\hat{S}_{0}^{(1)}(t) \hat{S}_{0}^{(1)}(0)\right\rangle=\left(\frac{1}{3}\right) S(S+1) \exp \left(-t / \tau_{S}\right), \\
& \left\langle\hat{S}_{ \pm 1}^{(1)}(t) \hat{S}_{\mp 1}^{(1)}(0)\right\rangle \\
& \quad=\left(-2^{-1}\right)(2 S+1)^{-1}
\end{aligned}
$$

$$
\begin{aligned}
T_{1, \text { dip }}^{-1}= & \frac{4 \gamma_{I}^{2} g^{2} \beta_{o}^{2}}{r^{6}}\left(\frac{\mu_{o}}{4 \pi}\right)^{2}\left\{\frac{S(S+1)}{3}\left[\left(\frac{2}{3}\right) \hat{C}_{0}^{(2)} \hat{C}_{0}^{(2)}-\widehat{C}_{+1}^{(2)} \hat{C}_{-1}^{(2)}\right] \cdot j\left(\omega_{I}\right)+2^{-1}\right. \\
& \times\left[6^{-1} \hat{C}_{0}^{(2)} \hat{C}_{0}^{(2)}-2^{-1} \hat{C}_{+1}^{(2)} \hat{C}_{-1}^{(2)}+\widehat{C}_{+2}^{(2)} \hat{C}_{-2}^{(2)}\right] \times(2 S+1)^{-1}\left|c_{S, m}^{+}\right|^{2}\left(j\left(\omega_{m}+\omega_{I}\right)+j\left(\omega_{m}-\omega_{I}\right)\right),
\end{aligned}
$$

where

$$
j(\omega)=\frac{\tau_{c}}{1+\left(\omega \tau_{c}\right)^{2}}
$$

and

$$
\left(\tau_{c}\right)^{-1}=\left(\tau_{S}\right)^{-1}+\left(\tau_{R}^{(1)}\right)^{-1} .
$$

In Eq. (19), the functions $\widehat{C}_{q}^{(2)}(\theta, \phi) \widehat{C}_{-q}^{(2)}(\theta, \phi) \mathrm{de}-$ scribe the orientation of the nuclear spin $\bar{I}$ in the molecular coordinate system. The explicit forms are

$$
\begin{aligned}
& \widehat{C}_{0}^{(2)}(\theta, \phi) \widehat{C}_{0}^{(2)}(\theta, \phi)=\frac{1}{4}\left(3 \cos ^{2} \theta-1\right)^{2}, \\
& \widehat{C}^{2}{ }_{+1}(\theta, \phi) \widehat{C}_{-1}^{2}(\theta, \phi)=-\frac{3}{2} \cos ^{2} \theta \sin ^{2} \theta, \\
& \widehat{C}_{+2}^{(2)}(\theta, \phi) \hat{C}_{-2}^{(2)}(\theta, \phi)=2^{-2} \sin ^{4} \theta .
\end{aligned}
$$

$$
\begin{aligned}
\left\langle\hat{S}_{+1}^{(1)}(t) \hat{S}_{-1}^{(1)}(0)\right\rangle+\left\langle\hat{S}_{-1}^{(1)}(t) \hat{S}_{+1}^{(1)}(0)\right\rangle & \\
= & \left(-2^{-1}\right)(2 S+1)^{-1} \exp \left(-t / \tau_{S}\right) \\
& \times \sum_{m}\left|c_{S, m}^{+}\right|^{2}\left(e^{i \omega_{m} t}+e^{-i \omega_{m^{t}}}\right)
\end{aligned}
$$

with $\omega_{m}=\widetilde{D}(2 m+1)$. These results have been simplified through the use of a single parameter $\tau_{S}=\tau_{m}^{(1)}=\tau_{m, m+1}^{(2)}$ to describe the relaxation of $\bar{S}$ in the molecular frame. Substituting Eqs. (18a)-(18c) into Eq. (12) and using Eqs. (6) and (11) gives, as the final result for the intramolecular dipolar paramagnetic relaxation increment,

$$
\begin{aligned}
T_{2, \mathrm{dip}}^{-1}= & \frac{4 \gamma_{I}^{2} g^{2} \beta_{o}^{2}}{r^{6}}\left(\frac{\mu_{o}}{4 \pi}\right)^{2}\left\{\frac{S(S+1)}{3}\right. \\
& \times\left[\left(\frac{2}{3}\right) \hat{C}_{0}^{(2)} \hat{C}_{0}^{(2)}-\widehat{C}_{+1}^{(2)} \hat{C}_{-1}^{(2)}\right] \cdot j(0)+2^{-1}\left[6^{-1} \widehat{C}_{0}^{(2)} \hat{C}_{0}^{(2)}-2^{-1} \hat{C}_{+1}^{(2)} \hat{C}_{-1}^{(2)}+\widehat{C}_{+2}^{(2)} \hat{C}_{-2}^{(2)}\right] \\
& \left.\times(2 S+1)^{-1} \sum_{m}\left|c_{S, m}^{+}\right|^{2}\left(j\left(\omega_{m}+\omega_{I}\right)+j\left(\omega_{m}-\omega_{I}\right)\right)\right\} .
\end{aligned}
$$

This result is identical to that for $T_{1, \mathrm{dip}}^{-1}$ except for the replacement of $j\left(\omega_{I}\right)$ by $j(0)$.

\section{B. Scalar relaxation}

The isotropic electron-nuclear scalar coupling is described by the Hamiltonian

$$
\begin{aligned}
H_{\mathrm{sc}} & =\hbar \mathscr{H}_{\mathrm{sc}}=\hbar A \bar{I} \cdot \bar{S} \\
& =\hbar A\left(I_{0}^{(1)} S_{0}^{(1)}-I_{+1}^{(1)} S_{-1}^{(1)}-I_{-1}^{(1)} S_{+1}^{(1)}\right) \\
& =\hbar \sum_{q}(-1)^{1-q 3^{-1 / 2}} F_{q}^{(1)} F_{-q}^{(1)}
\end{aligned}
$$

The fact that Eq. (19) is independent of the azimuthal angle $\phi$ results from the assumption of axial symmetry in $\mathscr{H}_{s}$.

The transverse relaxation rate can be calculated in a parallel manner. In terms of the components of $F_{q}^{(1)}, T_{2, \text { dip }}^{-1}$ can be written ${ }^{23}$

$$
\begin{aligned}
T_{2, \mathrm{dip}}^{-1}= & 2^{-1} \cdot 3^{-1} \int_{-\infty}^{\infty} d t\left[\left\langle F_{-1}^{(1)}(t) F_{+1}^{(1)}(0)\right\rangle e^{i \omega_{1} t}\right. \\
& +\left\langle F_{+1}^{(1)}(t) F_{-1}^{(1)}(0)\right\rangle e^{-i \omega_{t} t} \\
& \left.+\left\langle F_{0}^{(1)}(t) F_{0}^{(1)}(0)\right\rangle\right] .
\end{aligned}
$$

Transforming $S_{q}^{(1)}$ and $F_{q}^{(1)}$ to the molecular coordinate system with Eqs. (7) and (8) and calculating the time correlation functions $\left\langle F_{q}^{(1)}(t) F_{-q}^{(1)}(0)\right\rangle$ using Eqs. (9)-(18) gives 
enters the theory through the time correlation function,

$$
\overline{A(t) \cdot A(0)}=\overline{A^{2}} \exp \left(-t / \tau_{\text {ex }}\right)
$$

of the coupling constant. When $I$ and $S$ belong to the same molecule, $\tau_{\mathrm{ex}}$ is interpreted as a mean residence time in the paramagnetic molecule prior to a chemical exchange event. For intermolecular relaxation, $I$ and $S$ belong to different molecules, and $\tau_{\mathrm{ex}}$ can be interpreted as an effective contact time between solvent and solute, determined by the processes of translational diffusion. It is assumed that fluctuations in $A$ and $\bar{S}$ are uncorrelated in time and that both the spatial and spin variables remain at thermal equilibrium during the relaxation of $\bar{I}$. The general expressions for $T_{1, \mathrm{sc}}^{-1}$ and $T_{2, s c}^{-1}$ obtained from density matrix theory [Eqs. (6) and (23) ] can be written in the following form:

$$
\begin{aligned}
\frac{1}{T_{1, \mathrm{sc}}}= & -2^{-1} \int_{-\infty}^{\infty} \overline{A(t) \cdot A(0)}\left[\left\langle S_{-1}^{(1)}(t) S_{+1}^{(1)}(0)\right\rangle e^{i \omega_{I^{t}} t}\right. \\
& \left.+\left\langle S_{+1}^{(1)}(t) S_{-1}^{(1)}(0)\right\rangle e^{-i \omega_{r^{t}}}\right] d t \\
\frac{1}{T_{2, \mathrm{sc}}}= & 2^{-1} \int_{-\infty}^{\infty} \overline{A(t) \cdot A(0)}\left[\left(-2^{-1}\right)\right. \\
& \times\left\{\left\langle S_{-1}^{(1)}(t) S_{+1}^{(1)}(0)\right\rangle e^{i \omega_{0} t}\right. \\
& \left.+\left\langle S_{+1}^{(1)}(t) S_{-1}^{(1)}(0)\right\rangle e^{-i \omega_{r} t}\right\} \\
& \left.+\left\langle S_{0}^{(1)}(t) S_{0}^{(1)}(0)\right\rangle\right] d t .
\end{aligned}
$$

The spin variables in Eqs. (25)-(29) are written in the laboratory frame. As in the previous section, we assume that the electron spin Hamiltonian is dominated by a uniaxial zfs [Eq.(13)], which defines the quantization axes of $\bar{S}$. The transformation of the spin functions to the molecular frame and the calculation of the time correlation functions

$$
\begin{aligned}
\left\langle S_{+1}^{(1)}(t) S_{-1}^{(1)}(0)\right\rangle= & \sum_{q}\left\langle\hat{S}_{q}^{(1)}(t) \hat{S}_{-q}^{(1)}(0)\right\rangle \\
& \times \overline{\mathscr{D}}_{q+1}^{(1)}(t) \mathscr{D}_{-q-1}^{(1)}(0)
\end{aligned}
$$

was described above [Eqs. (14)-(18)]. As in the dipolar calculation, spin and spatial functions are assumed to be uncorrelated so that their averages can be computed separately. We arrive at the result

$$
\begin{aligned}
T_{1, \mathrm{sc}}^{-1}= & \frac{\overline{A^{2}}}{3}\left\{\frac{2}{3} S(S+1) j\left(\omega_{I}\right)+(2 S+1)^{-1}\right. \\
& \left.\times \sum_{m}\left|c_{S, m}^{+}\right|^{2}\left[j\left(\omega_{m}-\omega_{I}\right)+j\left(\omega_{m}+\omega_{I}\right)\right]\right\}, \\
T_{2, \mathrm{sc}}^{-1}= & \frac{\overline{A^{2}}}{3}\left\{\frac{2}{3} S(S+1) j(0)+(2 S+1)^{-1}\right. \\
& \left.\times \sum_{m}\left|c_{S, m}^{+}\right|^{2}\left[j\left(\omega_{m}-\omega_{I}\right)+j\left(\omega_{m}+\omega_{I}\right)\right]\right\},
\end{aligned}
$$

where

$$
\begin{aligned}
& \omega_{m}=\widetilde{D}(2 m+1), \\
& j(\omega)=\frac{\tau_{e}}{1+\omega^{2} \tau_{e}^{2}}
\end{aligned}
$$

with

$$
\tau_{e}^{-1}=\tau_{S}^{-1}+\tau_{\mathrm{ex}}^{-1}+\left(\tau_{R}^{(1)}\right)^{-1} .
$$

Expressions (31) and (32) differ from each other only in the factors $j\left(\omega_{I}\right)$ and $j(0)$.

\section{DISCUSSION}

For purposes of comparison, the Zeeman limit expressions for scalar relaxation corresponding to Eqs. (31) and (32) are $^{23}$

$$
\begin{aligned}
& T_{1, \mathrm{sc}}^{-1}=\frac{2}{3} A^{2} S(S+1) j_{2}\left(\omega_{S}-\omega_{I}\right), \\
& T_{2, \mathrm{sc}}^{-1}=\frac{1}{3} A^{2} S(S+1)\left[j_{2}\left(\omega_{S}-\omega_{I}\right)+j_{1}(0)\right] .
\end{aligned}
$$

The scalar correlation times are defined $\tau_{e, i}^{-1}=\tau_{S, i}^{-1}+\tau_{\mathrm{ex}}^{-1}$, where $i=1,2$ denote longitudinal and transverse relaxation times of $S$.

The expressions corresponding to the $\mathrm{zfs}$ limit [Eqs. (31) and (32)] and the Zeeman limit [Eqs. (35) and (36) ] coincide in the extreme narrowing limit. In this case, $\tau_{e}$ is short enough that $\omega_{I}, \omega_{S}, \omega_{m} \ll \tau_{e}^{-1}$, and hence

$$
j\left(\omega_{I}\right), j\left(\omega_{S}\right), j\left(\omega_{m}\right) \cong j(0) .
$$

With the use of the identity

$$
(2 S+1)^{-1} \sum_{m}\left|c_{S, m}^{+}\right|^{2}=\frac{2}{3} S(S+1)
$$

it is apparent that Eq. (31) reduces to Eq. (35), and Eq. (32) reduces to Eq. (36).

It is also possible to compare Eqs. (19) and (24) with the results of SBM theory, although only in a formal sense since the physical pictures in the two limits are quite different. The limiting expressions coincide in the extreme narrowing situation [Eq.(37)], when the geometric factors, $\widehat{C}_{q}^{(2)} \widehat{C}^{(2)}$, are averaged over the full space of the Euler angles. This average is given by the normalization relation

$$
\widehat{\widehat{C}_{q}^{(l)} \widehat{C}_{-q}^{(l)}}=(-1)^{q}(2 l+1)^{-1},
$$

with $l=2$. [This kind of averaging in Eq. (19) is clearly formal rather than physical.] With these replacements, Eq. (19) reduces to

$$
T_{1, \mathrm{dip}}^{-1}=\left(\frac{4}{3}\right)\left(\frac{\mu_{o}}{4 \pi}\right)^{2} \frac{\gamma_{1}^{2} g^{2} \beta_{o}^{2}}{r^{6}} S(S+1) j(0)
$$

which agrees with the SBM result in the extreme narrowing limit.

\section{Scalar reldxation in the zfs limit}

The most interesting difference between scalar relaxation in the Zeeman and $\mathrm{zfs}$ limits is in the relative magnitudes of $T_{1, \mathrm{sc}}^{-1}$ and $T_{2, \mathrm{sc}}^{-1}$. In the Zeeman limit, scalar coupling is frequently an important $T_{2}$ mechanism but rarely a significant contributor to $T_{1}$. This results from the fact that $j_{2}\left(\omega_{S}\right)$ is generally much smaller than $j_{1}(0)$, except in the unusual situation where $\left(\omega_{I}-\omega_{S}\right)^{2} \tau_{e}^{2}<1$, in which case both $T_{1, \mathrm{sc}}^{-1}$ and $T_{2, \mathrm{sc}}^{-1}$ are very small. In the zfs limit, in contrast, $T_{2, \mathrm{sc}}^{-1}$ and $T_{2, \mathrm{sc}}^{-1}$ are in most cases equal, with $T_{1, \mathrm{sc}}^{-1}$ much larger than predicted by SBM theory. Some aspects of this phe- 
nomenon have been described and commented upon by Benetis et al. ${ }^{10}$

The physical reason for this characteristic difference of the two limits is illustrated vectorially in Fig. 1. In the Zeeman limit [Fig.1(A)], $I$ and $S$ spins are shown as vectors precessing about the laboratory magnetic field $B$, with the time dependence of the spin components $I_{z}$ and $I_{ \pm}(t)$ given by the Heisenberg form of the operators,

$$
\begin{aligned}
& I_{+}(t)=e^{i \not \mathscr{H} z^{t}} I_{+} e^{-i \mathscr{H} z^{t}}=e^{+i \omega_{l} I_{Z^{t}} I_{+} e^{-i \omega_{I} I_{Z^{t}}}} \\
& =e^{+i \omega_{I} I_{Z} t} e^{-i \omega_{I}\left(I_{Z}-1\right) t} I_{+} \\
& \quad=e^{+i \omega_{I} t} I_{+} \\
& I_{-}(t)=e^{-i \omega_{I} t} I_{-} \\
& I_{Z}(t)=I_{Z}
\end{aligned}
$$

and similarly for $S_{ \pm}(t)$. (The commutation relations $\left[I_{ \pm}, I_{z}\right]=\mp I_{ \pm}$have been used to commute $I_{ \pm}$and $e^{-i \omega_{1} I_{z} t}$ in the first two relations). The $z$ components of $I$ and $S$ are static except for effects of relaxation. The relaxation of $I$ along the $z$ direction (i.e., $T_{1}$ relaxation) results from random fluctuations in the transverse components of the hyperfine energy, $2^{-1} \hbar A\left[I_{-}(t) \cdot S_{+}(t)+I_{+}(t) \cdot S_{-}(t)\right]$. These two terms oscillate at frequencies of $\pm\left(\omega_{S}-\omega_{I}\right)$, and it is the power density of the scalar interaction at these frequencies that is responsible for relaxation. In the case of $T_{2}$ processes, relaxation is effected by one transverse component and one longitudinal component of the hyperfine ener-

A
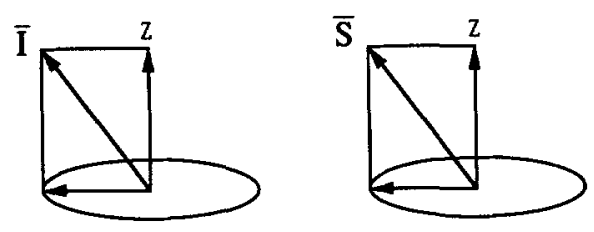

$\omega_{\text {I }}$

$\omega_{\mathrm{s}}$
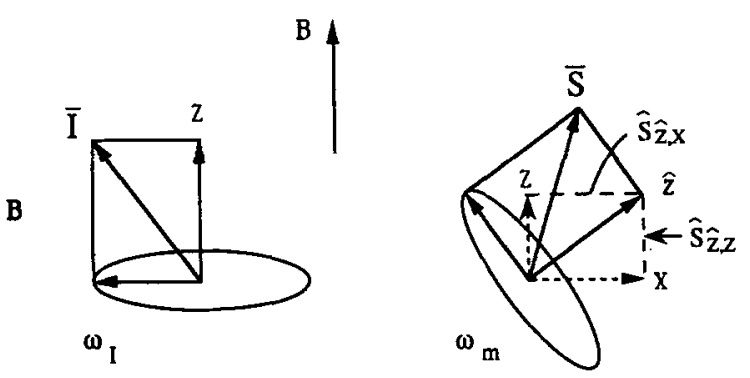

FIG. 1. Scalar coupling in the Zeeman (A) and zfs (B) limits. In the Zeeman limit (A), spins $\bar{I}$ and $\bar{S}$ are quantized by the external field $B$, about which they precess with angular frequencies $\omega_{I}$ and $\omega_{S}$. In the zfs limit (B), $\bar{I}$ is quantized by the external field, and $\bar{S}$ is quantized along the molecular $\hat{z}$ axis, about which it precesses with angular frequency $\omega_{m} \cdot \hat{S}_{\hat{z}, x}$ and $\hat{S}_{\bar{z}, z}$ are the components of $\bar{S}$ that are static (i.e., non-oscillating) along the laboratory $x$ and $z$ axes, respectively. These give rise to the low frequency portions of the spectral density function of scalar coupling. gy, and the corresponding spectral density functions are $j(0)$ and $j\left(\omega_{S}-\omega_{I}\right)$.

The situation in the zfs limit is pictured in Fig. 1(B). As in the Zeeman limit, relaxation of $I_{z}$ is driven by fluctuations in $S_{x}(t)$ and $S_{y}(t)$ (i.e., the transverse components of $S$ in the laboratory frame). Relaxation of $I_{x}$ is driven by the components $S_{y}(t)$ and $S_{z}(t)$ (also in the laboratory frame). In this limit, however, $\bar{S}$ is quantized in the molecular frame $(\hat{x}$, $\hat{y}, \hat{z})$. The hyperfine field associated with the $\hat{z}$ component of $\bar{S}, \hat{S}_{\hat{z}}$, is non-oscillating. $\hat{S}_{\hat{z}}$ has components along the laboratory $x$ and $z$ directions, denoted $\hat{S}_{\hat{z}, x}$ and $\hat{S}_{\hat{z}, z}$ in Fig. 1(B). The longitudinal interaction between $\hat{S}_{\bar{z}, z}$ and $I_{z}$ is static, while the transverse interaction between $\hat{S}_{\hat{z}, x}$ and $I_{x}$ oscillates with frequency $\omega_{I}$. The former contributes to $T_{2}$, the latter to $T_{1}$. These are the low-frequency relaxation contributions that are described, respectively, by $j(0)$ and $j\left(\omega_{I}\right)$ in Eqs. (8) and (7). The spin components $\hat{S}_{\hat{x}}$ and $\hat{S}_{\hat{y}}$ that are transverse in the molecular frame oscillate at the transition frequencies $\omega_{m}$ and give rise to the terms containing $j\left(\omega_{m} \pm \omega_{I}\right)$. However, it is the low-frequency terms [at $j(0)$ and $\left.j\left(\omega_{I}\right)\right]$ that will dominate $T_{1, \mathrm{sc}}^{-1}$ and $T_{2, \mathrm{sc}}^{-1}$ in nearly all circumstances.

Another noteworthy point is the fact that scalar relaxation depends upon molecular reorientation in the $\mathrm{zfs}$ limit, but not in the Zeeman limit. The scalar interaction $A \bar{I} \cdot \bar{S}$ is independent of the spatial orientation of the interspin vector. However, it is not independent of changes in the relative orientation of the coordinate systems in which $I$ and $S$ are quantized, hence the dependence of $T_{1, \mathrm{sc}}^{-1}$ and $T_{2, \mathrm{sc}}^{-1}$ upon $\tau_{R}^{(1)}$ in Eqs. (31) and (32). As is the case for dipolar relaxation in the $\mathrm{zfs}$ limit, the relevant reorientational correlation time is $\tau_{R}^{(1)}$ rather than $\tau_{R}^{(2)}$.

\section{CONCLUSIONS}

The dipolar and scalar increments to the nuclear spin relaxation rates are described in the zfs limit by Eqs. (19), (24), and (31), (32), respectively. These expressions are valid in the limit where the electron spin Hamiltonian is dominated by a quadratic $\mathrm{zfs}$ with uniaxial symmetry. The inclusion of first-order Zeeman couplings in the analysis is fairly straightforward ${ }^{20,21}$ although we have not done so here.

The analysis assumes that the electron spin relaxation functions are exponential in time. This assumption is strictly true in the Redfield limit, ${ }^{25-27}$ i.e., when the motional correlation times that produce electron spin relaxation are short compared to $\tau_{s}$. It is frequently stated in the literature that the spin system is outside the Redfield limit when $\tau_{S}$ is comparable to or shorter than $\tau_{R}$. However, this criterion is probably unreliable when the $\mathrm{zfs}$ is large. In the $\mathrm{zfs}$ limit, the relaxation of $S$ is not produced by reorientational modulation of the zfs interaction, but rather by reorientational modulation of the Zeeman coupling and/or by vibrational modulation of the $\mathrm{zfs}$ tensor. Which of these mechanisms dominates in a particular situation is not clear a priori. The Zeeman mechanism is field dependent (as the square of the field strength), while the zfs mechanism depends on the gradients of the $\mathrm{zfs}$ tensor elements with respect to the normal 
coordinates of the asymmetric vibrational modes. ${ }^{28}$ For vibrational modulation of the $\mathrm{zfs}$ tensor, the characteristic correlation times are those for vibrational damping $\left(\tau_{v}\right)$, of the order of a few picoseconds. In this case, an exponential relaxation function should be appropriate for quite short electron spin relaxation times (say, $>10 \mathrm{ps}$ ). When the Zeeman relaxation mechanism dominates, the relevant relaxation-producing motions are due to molecular reorientation, and the correlation times are relatively long $\left(\tau_{R} \geqslant 30 \mathrm{ps}\right)$. In this case, the Redfield limit is invalid and the use of an exponential relaxation function in Eq. (17) is questionable.

It seems likely, however, that for systems with large zfs the vibrational relaxation mechanism will normally dominate, since for ions with large static zfs, the gradients of the zfs are likewise expected to be large. Extending this line of reasoning, one would predict that electron spin relaxation times for ions in the zfs limit will be independent of $\tau_{R}$ but not of $\tau_{v}$. This point is readily checked from the temperature dependence of $\tau_{s}$, which is rather different for the processes of molecular reorientation and vibrational damping. Molecular reorientation is described (at least approximately) by the Debye equation, which predicts that $\tau_{R}$ varies as (viscosity over temperature). The vibrational damping mechanism $^{28}$ leads to a temperature dependence, $\tau_{S}^{-1} \propto T^{1 / 2} \operatorname{coth}\left(\hbar \omega_{0} / k^{\prime}\right)$, where $\omega_{0}$ are characteristic fundamental vibrational frequencies of atoms in the first coordination sphere of the metal. The predicted temperature dependence for the vibrational mechanism is considerably milder than for reorientation. A recent study involving $\mathrm{Mn}$ (III) $[a c a c]_{3}$ in the $\mathrm{zfs} \mathrm{limit}^{21}$ indicated that in this case the dominant $\tau_{S}$ relaxation mechanism is in fact vibrational. For chemical systems in the zfs limit with very long $\tau_{R}$ (e.g., metallo-enzymes, transition metal ions bound to polymers), the Zeeman interaction is effectively static and relaxation of $S$ will almost certainly be dominated by vibrational motions of the ligands. It is probably significant in this regard that the spin relaxation times for $\mathrm{Cr}$ (III) are essentially the same in aqueous solutions of the low molecular weight binary nucleotide complexes $\mathrm{Cr}$ (III) [ADP] and $\mathrm{Cr}$ (III) [ATP] as in high molecular weight ternary complexes in which these binary complexes ( $\mathrm{Cr}$ (III) [ADP], $\mathrm{Cr}$ (III) [ATP]) bind to metallo-enzymes. ${ }^{29,30}$

\section{ACKNOWLEDGMENT}

This work was supported by the U. S. Department of Agriculture in the form of research grants (Nos. 87 CRCR1-2344 and C/89-CRCR-2344).

${ }^{1}$ M. Guéron, J. Magn. Reson. 19, 58 (1975).

${ }^{2}$ I. Solomon, Phys. Rev. 99, 559 (1955).

${ }^{3}$ N. Bloembergen and L. O. Morgan, J. Chem. Phys. 34, 842 (1961).

${ }^{4}$ D. R. Burton, S. Forsèn, G. Karlstrom, and R. A. Dwek, Prog. Nucl. Magn. Reson. Spectrosc. 13, 1 (1980).

${ }^{5}$ N. Benetis, J. Kowalewski, L. Nordenskiöld, H. Wennerström, and P.-O. Westlund, Mol. Phys. 48, 329 (1983).

${ }^{6} \mathrm{~A}$. Abragam and B. Bleaney, Electron Paramagnetic Resonance of Transition Ions (Clarendon, Oxford, 1970).

${ }^{7}$ R. M. Lynden-Bell, Mol. Phys. 22, 837 (1971).

${ }^{8}$ J. Freed, G. V. Bruno, and C. Polnaszek, J. Chem. Phys. 55, 5270 (1971)

${ }^{9}$ N. Benetis, J. Kowalewski, L. Nordenskiöld, H. Wennerström, and P.-O. Westlund, Mol. Phys. 50, 515 (1983).

${ }^{10}$ N. Benetis, J. Kowalewski, L. Nordenskiöld, H. Wennerström, and P.-O. Westlund, J. Magn. Reson. 58, 261 (1984).

"' P.-O. Westlund, H. Wennerström, L. Nordenskiöld, J. Kowalewski, and N. Benetis, J. Magn. Reson. 59, 91 (1984).

${ }^{12}$ S. Szymanski, A. M. Gryff-Keller, and G. Binsch, J. Magn. Reson. 68, 399 (1986).

${ }^{13}$ L. -P. Hwang, Mol. Phys. 49, 1341 (1983).

${ }^{14}$ T. -H. R. Chen, S-J. Den, and L.-P. Hwang, Proc. Natl. Sci. Council (Rep. of China) 8, 224 (1984).

${ }^{15}$ L. -P. Hwang and C.-Y. Ju, J. Chem. Phys. 83, 3775 (1985).

${ }^{16}$ H. L: Friedman, M. Holz, and H. G. Hertz, J. Chem. Phys. 70, 3369 (1979).

${ }^{17}$ N. Benetis, J. Kowalewski, L. Nordenskiöld, and U. Edlund, J. Magn. Reson. 58, 282 (1984).

${ }^{18}$ P. -O. Westlund, N. Benetis, and H. Wennerström, Mol. Phys. 61, 177 (1987).

${ }^{19}$ P. - L. Wang, J.-H. Lee, S.-M. Huang, and L.-P. Hwang, J. Magn. Reson. 73, 277 (1987).

${ }^{20}$ U. Lindner, Ann. Phys. (Leipzig) 16, 319 (1965).

${ }^{21}$ T. Bayburt and R. R. Sharp, J. Chem. Phys. 92, 5892 (1990).

${ }^{22}$ B. L. Silver, Irreducible Tensor Methods (Academic, New York, 1976).

${ }^{23}$ A. Abragam, The Principles of Nuclear Magnetism (Oxford University, Oxford, 1961), Chap. VIII.

${ }^{24}$ W. T. Huntress, Adv. Nucl. Magn. Res. Spectrosc. 4, 2 (1970).

${ }^{25}$ A. K. Gregson, D. M. Doddrell, and D. T. Pegg, Aust. J. Chem. 31, 469 (1978).

${ }^{26}$ D. T. Pegg and D. M. Doddrell, Aust. J. Chem. 29, 1869 (1976).

${ }^{27}$ A. D. McLachlan, Proc. R. Soc. London Ser. A 280, 27 (1964).

${ }^{28}$ S. A. Al'tshuler and K. A. Valiev, Sov. Phys. JETP 35, 661 (1959).

${ }^{29}$ M. Villafranca, Methods Enzymol. 87, 180 (1982).

${ }^{30}$ M. S. Balakrishnan and J. Villafranca, Biochemistry 17, 3531 (1978). 